\title{
The old antibiotic colistin: an update on its use in children
}

\author{
Sukhbir K. Shahid ${ }^{1}$ \\ ${ }^{1}$ Consultant Pediatrician and Neonatologist, Mumbai, India
}

*Corresponding Author: Dr. Sukhbir K. Shahid, Consultant Pediatrician and Neonatologist, Shahid Clinic, Ghatkopar (E), Mumbai400077 MH India, Phone: 0091-9869036606, Email: s_kaur_shahid@yahoo.com

\section{Abstract}

Colistin is an old antibiotic which has rekindled the interest of infectious doctors worldwide. It is a polypeptide antibiotic belonging to the polymyxin class of antibiotics. It was discovered in 1947 and clinicians started using it for gram-negative infections. But its main drawback of nephrotoxicity and availability of better and safe antibiotics led to its use getting obsolete. But when the bacteria showed alarming resistance to the higher antibiotics, colistin was revisited for such antimicrobial resistant strains of bacteria. Efficacy of colistin against multi-drug resistant bacteria makes it an antibiotic of choice in intensive care setups. It is used as a last-line in these severe life-threatening infections in adults and children. Despite having been known for over seventy years now, there are significant lacunae in knowledge on its pharmacokinetics, pharmacodynamics, and correct dosing. It also has the problem of variable labelling by manufacturers and resistance is also rising steadily to this antibiotic. Research is ongoing to collect more scientific data on its mechanism of action and pharmacokinetics but more studies are needed especially in children to be able to extend its use in them. Colistin does hold promise in the world of infections to tackle resistant nosocomial infections and prevent emergence of superbugs.

Keywords: Colistin, Old Antibiotic, Renewed Uses, Nosocomial Infections, Multi-Drug Resistant Infections.

\section{INTRODUCTION}

Medical fraternity is increasingly encountering bacteria that are resistant to conventional mainstream antibiotics. Most of these infections are hospital-acquired but community-acquired resistant bugs are also on the rise ${ }^{[1]}$. Children admitted in pediatric wards and intensive-care setups are not spared of it. These infections lead to increased morbidity and mortality in them ${ }^{[2]}$. Pediatric intensivists find it hard to manage these lifethreatening infections. Research into improved antibiotics against such resistant nosocomial infections is limited and has not met with much success ${ }^{[3]}$. Often, clinicians fall back on the old antibiotic, colistin that is often used in such cases as a last resort ${ }^{[4]}$. But resistance of these superbugs to colistin is also seen these days. What is the current status of colistin in managing such highly-resistant bacterial infections in children? Are there any further options if colistin is ineffective in such cases? What is the future of this antibiotic in the infectious world? All this and more forms the purview of this article.

\section{NOSOCOMIAL INFECTION ANTIBIOTICS RESISTANCE}

Antimicrobial resistance (AMR) is steadily rising in the world. Antibiotics which seemed to work around two decades back in the management of bacteria such as E. Coli, Pseudomonas aeruginosa, Acinetobacter sp., and klebsiella pneumoniae are failing to clear these pathogenic organisms from the body. An invisible pandemic is creeping in and this would threaten future patient care and compromise the health care sector ${ }^{[5]}$.

An Egyptian University Hospital study of 2016 revealed that nosocomial infections were present in nearly $20 \%$ of their hospitalized children. These were multi- and pan-drug-resistant infections and led to treatment failures and four-fold rise in mortality ${ }^{[6]}$. The current coronavirus pandemic has pushed antimicrobial resistance upwards due to indiscriminate use of antibiotics at many centers of the world ${ }^{[7]}$. 
$\mathrm{WHO}$ has stated that antimicrobial resistance is in the top 10 list of global public health threats that affects living beings [8]. Irrational antibiotics usage is the key player in development of such multi-drug resistant (MDR) pathogens with associated enhanced morbidity and mortality ${ }^{[8]}$. While these infections are demanding newer better-efficacy antibiotics, unfortunately, the pipeline of such antibiotics against these dreadful infections is relatively dry. These difficult-to-treat infections also led to prolonged hospital stays, human productivity and economic losses, increased expenditure, and strain the health care system. This could irreversibly impact crucial areas such as cancer chemotherapy, transplant medicine, and major surgeries [8].

Prevalence of ciprofloxacin-resistant E. Coli is as high as $92.9 \%$ in some centers [8]. And this quinolone antibiotic is not useful against $79.4 \%$ of Klebsiella pneumoniae. Methicillin-resistant staphylococcus aureus (MRSA) form a significant number of infective cases in children and more than half of such cases succumb to it ${ }^{[8]}$. All these resistant pathogens also increasingly demonstrate resistance to carbapenem, meropenem, ceftaxidime, and cefepime. In such MDR cases, colistin comes as a salvageable antibiotic.

\section{COLISTIN-AN OVERVIEW}

\section{Introduction}

Colistin (polymyxin E) is a cyclic nonribosomal polycationic decapeptide that belongs to polymyxin groups of antibiotics. The other polymyxin antibiotic used is polymyxin B. The hydrophilic and the lipophilic moieties of colistin provide it with its bactericidal potency. It acts best against gram-negative organisms that are resistant to other antibiotics and forms a lastline antibiotic therapy against such microbes. Discovered over seven decades back in 1947, this antibiotic produced by grampositive bacteria of the genus Paenibacillus was used in oral form for infantile gut infections and in inhaled form for infective lung conditions. Its topical preparations were used in skin creams and parenteral preparations were given to manage systemic infections. It has also been administered intrathecally and intraventricularly for local actions [9]. However, its use fell in 1980s when antibiotics with better renal safety profile were discovered. But the advancing global pandemic of resistance of hospital bugs to the higher generation antibiotics forced clinicians to reconsider and redeploy colistin as therapy for such resistant cases. This led to re-inclusion of colistin in the therapeutic armamentarium for such infections both in adults as well as children [10]. Susceptibility of these resistant bacteria to colistin is largely preserved though rising instances of resistance to it are also seen lately.

\section{Spectrum of bactericidal activity and mechanism of action}

Colistin has a narrow antibacterial activity spectrum. Bacteria against which colistin is efficacious include Acinetobacter baumannii, klebsiella pneumoniae, carbapenem-resistant Enterobacteriaceae, pseudomonas aeruginosa and the like. It also has potency against Stenotrophomonas maltophilia, salmonella sp., shigella sp., H. Influenza, Bordetella pertussis, and Legionella pneumophilia ${ }^{[11]}$.

Colistin has a fast and concentration-dependent action on the susceptible organisms and demonstrates post-antibiotic and inoculum effects ${ }^{[12]}$. It acts basically on the lipopolysaccharides (LPS) of outer membrane of the bacteria. Colistin displaces the divalent cations such as calcium and magnesium in the negative-charged phosphate of lipid A of LPS and this action is bactericidal [13]. Its hydrophobic end has a detergent-like action on both the outer and inner membranes of the pathogen. A recent study from Imperial College, London has shown that the experimental antibiotic murepavadin can trigger LPS accumulation in the inner membrane of the bacteria and thus sensitize it to the effect of colistin. Thus, it can eradicate PS. aeruginosa from the lungs rapidly and effectively ${ }^{[14]}$. Colistin can also penetrate biofilms of Pseudomonas aeruginosa in lungs of cystic fibrosis patients. These biofilms have an oxygen-deprived milieu and colistin works best in such an environment ${ }^{[15]}$.

\section{Pharmacokinetics and pharmacodynamics}

Since colistin is unabsorbed from the gastrointestinal tract, parenteral administration is needed for systemic effects. For this, the inactive prodrug form of colistin called colistimethate sodium is used. This is hydrolyzed in the body to its active metabolite, colistin. Though it is an old antibiotic, knowledge on its pharmacokinetics is limited. It has a complicated pharmacokinetics with a lot of inter-patient variability and a very narrow therapeutic index [16]. The drug gets concentrated in the heart, muscles, kidney, and liver after intravenous administration. But drug concentrations in pleura, lungs, CSF, and bones are found to be lesser than the therapeutic requirements [17]. The half-life of the drug is 14.4 hours and the predicted maximum plasma concentration in a steady state is $2.3 \mathrm{mg} / \mathrm{L}$. But critically ill patients may need a loading dose to reach a therapeutic concentration in the blood [18]. It is eliminated from the body by kidneys as well as by non-renal mechanisms [12].

\section{Dosing and issues}

Due to the gaps in knowledge of pharmacology of colistin, its use is far from optimal. There are no recommendations on its dosages and hence there is no uniformity among physicians on its effective and safe dosing that could help prevent resistance development. There is lack of scientific data on its ideal dosing in critically-ill patients with impaired renal functions or in cystic fibrosis cases [19]. Variable concentration packaging and labelling by manufacturers has further added to the confusion. Some label it in international units while others put it as milligrams of colistin base or colistimethate sodium (CMS). Generally, $80 \mathrm{mg}$ of CMS is equivalent to 100000 units or $30 \mathrm{mg}$ of colistin base activity. But it becomes difficult to prescribe since there is neither a proper standardization of dosing nor any regulation related to it. The usual doses used are 2.5 to $5 \mathrm{mg} / \mathrm{kg}$ of colistin base per day or 6 to $12 \mathrm{mg} / \mathrm{kg}$ of CMS per day. In cases of colistin as aerosol or in nebulized form, the dose employed is 1-2 million units two times a day. 
There are some isolated studies done in the last two decades that have revealed that protein-binding of polymyxin $B$ depends on plasma concentration of the acute phase reactant, $\alpha_{1}$-acid glycoprotein ${ }^{20]}$. Since in infected patients, $\boldsymbol{\alpha}_{1}$-acid glycoprotein is higher, they have higher protein-binding of colistin (78.5$92.4 \%$ ). Hence the proportion of unbound polymyxin B falls below the minimum inhibitory concentration required to kill the pathogen. This also holds true for colistin and hence dosing needs to be reviewed in such gravely-sick patients [21].

\section{Toxicity}

Another area of concern for colistin has been its safety. The major side-effects reported are nephrotoxicity and neurotoxicity. In around 3.5-58\% of the cases, deranged renal functions are noticed. These are directly proportional to the doses and frequency used [22, 23]. Neurotoxicity is reported less often and around 0 to $7 \%$ suffers from it ${ }^{24-26]}$. It also has a propensity to lead to anaphylactic reaction in some individuals, muscular weakness, and Clostridium difficile-associated diarrhoea. When used as inhalational therapy, it could lead to bronchial constriction ${ }^{[27]}$. It be noted that aerosolized form of CMS is not FDA approved ${ }^{[28]}$.

\section{Synergistic actions with other antibiotics}

Concomitant administration of other anti-microbials such as rifampicin, anti-pseudomonal antibiotics, doxycycline, minocycline, and azithromycin with colistin has a synergistic effect with improved outcomes in patients. Older studies reveal that its combination with drugs such as trimethoprim broadens its effective antibacterial spectrum and also prevents resistance to trimethoprim-sulfamethoxazole [29]. Murepavadin is still experimental buts its concomitant use with colistin has synergism [14]. Once murepavadin enters clinical trials, its combination with colistin would be studied to determine its efficacy and safety in adults and children ${ }^{[14]}$.

\section{Colistin resistance}

Due to less use, resistance to colistin is rare but is showing a rise. Plasmid-located colistin-resistant gene called $\mathrm{mcr}-1$ (mobilized colistin resistance-1 gene) was first discovered in China in 2011. Later, it was also confirmed in other countries of the world. It modifies LPS structure that leads to inhibition of binding of colistin to it [30]. This remains the predominate mode of transferable colistin resistance in bacteria but chromosomemediated drug resistance also occurs ${ }^{[31]}$. The suboptimal dosing utilized due to lack of adequate pharmaokinetics knowledge also has a role to play in the development of colistin resistance ${ }^{[32]}$. Since the drug is used relatively less often, there is no standard method to gauge resistance to colistin in the laboratory. The French Microbiological Society has decided on a cut-off of 2 $\mathrm{mg} / \mathrm{L}$ for labelling resistance. The British Society for Antimicrobial Chemotherapy states that $4 \mathrm{mg} / \mathrm{L}$ or less is considered as sensitive and $8 \mathrm{mg} / \mathrm{L}$ or more would be resistance ${ }^{\text {[33] }}$. Some bacterial strains demonstrate inherent and variable resistance to colistin ${ }^{[34]}$.

\section{COLISTIN IN CHILDREN: CURRENT SCENARIO}

Colistin was used in children of cystic fibrosis for many years, and since last decade, paediatricians have extended this use to treat even non-CF infections ${ }^{[35]}$. The antibiotic is used in children infected with hard-to-manage resistant strains of gram-negative bacteria when other higher antibiotics fail to eradicate the superbug. Though there are very limited studies in children data is accumulating and it is now known that colistin is efficacious and safe in children ${ }^{[35-38]}$. Colistin has been employed for a wide range of indications in nosocomial infections in children such as pneumonias including ventilator-associated pneumonia, meningitis, ventriculitis, skin, bone, soft tissue infections, urinary tract infections, and sepsis.

A multi-centric pediatric study in the USA showed that colistin was prescribed in $67.4 \%$ of multidrug-resistant Pseudomonas, $11.9 \%$ of multidrug-resistant Acinetobacter baumanii, $13.0 \%$ of carbapenemase-producing Enterobacteriaceae and $5.4 \%$ of extended-spectrum $\beta$-lactamase producing Enterobacteriaceae. The antibiotic maintained potent activity against nearly $4 / 5^{\text {th }}$ of the infections. But in more than one-fifth of the cases $(20.5 \%)$, the bacteria showed resistance to colistin as well. $22 \%$ of these pediatric patients had nephrotoxicity with use of colistin but fortunately the kidney function tests returned to baseline on stoppage of the incriminating antibiotic. This renal toxicity was observed more in older children $\geq 13$ years of age. 4 out of 92 children had neurotoxicity that was reversible ${ }^{[37]}$. Another study on children admitted in a PICU of a University hospital in Turkey showed that there was a favourable outcome in $68.3 \%$ of the infected children. The researchers advised against use of other nephrotoxic drugs along with colistin to prevent renal damage [38].

In children, similar to adults, there is confusion regarding the doses to be used. Expression of colistin concentration has been in one of these three ways: international units of CMS, mgs of CMS, and mgs of colistin base activity. The conversion factor of these three is similar as in adults. Most children are given colistin in doses of $2.5 \mathrm{mg} / \mathrm{kg}$ every 12 hours (range 1.35 to 5/kg/dose). European Medicines Agency recommends a dosing regimen of $75000-150000 \quad \mathrm{IU} / \mathrm{kg} /$ day. And US Food and Drug Administration states that colistin should be administered in dose of $83,000-166,000 \mathrm{IU} / \mathrm{kg} / \mathrm{d}$ (that is $2.5-5 \mathrm{mg} / \mathrm{kg} / \mathrm{d}$ of colistin base in two to four divided doses) ${ }^{[39]}$.

The pharmacokinetic data in children and newborns is largely missing. But from the limited studies, it is recommended that higher doses may be required in infections where the MIC is more than 1 microgram/L in isolates from neonates and young children ${ }^{[40]}$. Also, intrathecal and intraventricular administration is advised in meningitis in children since level of colistin reached in CSF with regular doses is insufficient to eradicate the bacteria. Nebulized CMS in dose of $120000 \mathrm{IU} / \mathrm{kg}$ was found to be adequate to have the desired effect ${ }^{[41]}$. 
Incidence of kidney toxicity was in the range of 3 to $10 \%$ in children. But in the multicentric study from the USA cited above, it was as high as $22 \%{ }^{[37]}$. And reversible neurotoxicity in the form of headaches and paresthesia though rare does occur [42]. In neonates, electrolyte disturbances in the form of hypomagnesemia and hypokalemia have also been noticed and require supplementation and regular serum electrolyte level monitoring $[43,44]$.

\section{CONCLUSION}

Colistin is needed to tackle resistant cases of nosocomial infections. Though research on it is scattered and incomplete, it has proven to be a good antibiotic which physicians can bank on for treating such dreadful bugs. This salvage antibiotic is also useful in children who tolerate it well with none or minimal reversible side-effects. The antibiotic has stood the test of time and renewed interest in it has found it to be useful in treating resistant bugs. But there is a demand for more studies in understanding its pharmacokinetics and pharmacodynamics in order to harness its potential benefits to the greatest extent.

Drug resistance is a global public health problem that is rising despite efforts to curtail it. It needs coordinated and focused multidisciplinary efforts to arrest its further rise. All those who are engaged in human, animal health, plant health and environment including the food manufacturers and distributors need to get together and work towards a common goal of minimizing the irrational use of antibiotics on this planet. Policies need to be made in that direction and enforced. Besides, research should be directed to expand the antibiotic pipeline. This should also be for development of certain vaccines and better diagnostic tools towards these superbugs. One Health programs and World Antimicrobial Awareness Week campaign are steps targeting this menace. Emergence of resistance to antifungals, antiparasitic drugs and antivirals should also be aimed to be prevented. The threat is real and needs to be handled wisely and aggressively.

\section{REFERENCES}

1. Van Duin D, Paterson DL. Multi-drug resistant bacteria in the community: An update. Infect Dis Clin North Am. 2020; 34(4):709722

2. Shi J, Sun T, Cui Y, Wang C, Wang F, Zhou $Y$ et al. Multi-drug resistant and extensively multi-drug resistant Acinetobacter baumannii hospital infection associated with high mortality: a retrospective study in the pediatric intensive care unit. BMC Infect Dis 2020; 20(1):597. Available from https://bmcinfectdis.biomedcentral.com/articles/10.1186/s12879020-05321-y

3. Stephens LJ, Werrett Mv, Sedgwick AC, Bull SD, Andrews PC. Antimicrobial innovation: a current update and perspective on the antibiotic drug development pipeline. Future Med Chem. 2020; 12(22):2035-2065.

4. El-Sayed AMAE, Zhong LL, Shen C, Yang Y, Doi Y, Tian GB. Colistin and its role in the era of antibiotic resistance: an extended review (2000-2019). Emerg Microbes Infect. 2020; 9(1):868-885.

5. Nieuwlaat R, Mbuagbaw L, Mertz D, Burrows LL, Bowdish DME, Moja L et al. Coronavirus disease 2019 and antimicrobial resistance: parallel and interacting health emergencies. Clin Infect Dis. 2021; 72(9):1657-1659.
6. El-Nawawy A, Ashraf GA, Antonios MAM, Meheissen MA, El-Alfy MMR. Incidence of Multidrug-Resistant Organism Among Children Admitted to Pediatric Intensive Care Unit in a Developing Country. Microb Drug Resist. 2018; 24(8):1198-1206.

7. Ansari S, Hays JP, Kemp A, Okechukwu R, Murugaiyan J, Ekwanzala MD et al. The potential impact of the COVID-19 pandemic on global antimicrobial and biocide resistance: an AMR Insights global perspective. JAC Antimicrobial Resist. 2021; 3(2):dlab038. Available from https://www.ncbi.nlm.nih.gov/pmc/articles/PMC8083476/

8. Information downloaded on $10^{\text {th }}$ August 2021 from https://www.who.int/news-room/fact-sheets/detail/antimicrobialresistance

9. Iaria C, Giammalva GR, Spicola D, Fimognari AM, Imburgia C, Di Lorenzo $\mathrm{F}$ et al. Multi-drug-resistant gram-negative postneurosurgical meningitis and the role of intraventricular colistin: a case series. Infez Med 2021; 29(1):130-137.

10. Colistimethate Sodium Monograph for Professionals. Drugs.com Retrieved on $8^{\text {th }} \quad$ August 2021 from https://www.drugs.com/monograph/colistimethate.html

11. Lim LM, Ly N, Anderson D, Yang JC, Macander L, Jarkowski A 3rd et al. Resurgence of Colistin: A Review of resistance, toxicity, pharmacodynamics, and dosing. Pharmacotherapy. 2010; 30(12):1279-1291.

12. Zhu Y, Monsel A, Roberts JA, Pontikis K, Momoz O, Rello J et al. Nebulized colistin in ventilator-associated pneumonia and tracheobronchitis Historical backgroaund, pharmacokinetics and perspectives Microorganisms 2021; 9(6):1154, 25 pages. Available from https://www.mdpi.com

13. Hancock RE. Peptide antibiotics. Lancet. 1997; 349:418-422.

14. Sabnis A, Hagart KL, Klockner A, Becce M, Evans LE, Furniss $\mathrm{RCD}$ et al. Colistin kills bacteria by targeting lipopolysaccharide in the cytoplasmic membrane. Elife 2021; 10: e65836. Available from https://doi.org/10.5061/dryad.98sf7m0hh

15. Kolpen M, Appeldorff CF, Brandt S, Mousavi N, Kragh KN, Aydogan $S$ et al. Increased bactericidal activity of colistin on Pseudomonas aeruginosa bioflims in anaerobic conditions. Pathog Dis 2016; 74(1):ftv086. Available from https://pubmed.ncbi.nlm.nih.gov/26458402/

16. Thien BT, Velkov T, Nation RL, Forrest A, Tsuji BT, Bergen PJ, Li J. Pharmacokinetics/pharmacodynamics of colistin and polymyxin B: are we there yet? Int. J. Antimicrobial Agents. 2016; 48(6):592597.

17. Gurjar M. Colistin for lung infection: an update. J Intensive Care. 2015; 3:3. Available from https://jintensivecare.biomedcentral.com/articles/10.1186/s40560 $-015-0072-9$

18. Plachouras D, Karvanen M, Friberg LE, Papadomichelakis E, Antoniadou A, Tsangaris I et al. Population pharmacokinetic analysis of colistin methanesulfonate and colistin after intravenous administration in critically ill patients with infections caused by gram-negative bacteria. Antimicrob Agents Chemother. 2009; 53:3430-3436.

19. Nation RL, Li J. Colistin in the $21^{\text {st }}$ century. Curr Opinion in infectious diseases 2009; 22(6):535-543.

20. Zavascki AP, Goldani LZ, Cao G, Superti SV, Lutz L, Barth AL et al. Pharmacokinetics of intravenous polymyxin B in critically-ill patients. Clin Infect Dis. 2008; 47:1298-1304.

21. Michalopoulos AS, Falagas ME. Colistin: recent data on pharmacodynamics properties and clinical efficacy in critically ill patients. Ann. Intensive Care 2011; 1:30. https:// doi.org/10.1186/2110-5820-1-30

22. Wallace SJ, Li J, Nation RL, Rayner CR, Taylor D, Middleton D et al. Subacute toxicity of colistin methanesulfonate in rats: comparison of various intravenous dosage regimens. Antimicrob Agents Chemother. 2008; 52:1159-1161.

23. Lewis JR, Lewis SA. Colistin interactions with the mammalian 
urothelium. Am J Physiol Cell Physiol. 2004; 286:C913-C922.

24. Pogue JM, Lee J, Marchaim D, Yee V, Zhao JJ, Chopra T et al. Incidence of and risk factors for colistin-associated nephrotoxicity in a large academic health system. Clin Infect Dis. 2011; 53:879884.

25. Doshi NM, Mount KL, Murphy CV. Nephrotoxicity associated with intravenous colistin in critically ill patients. Pharmacotherapy. 2011; 31:1257-1264.

26. Hartzell JD, Neff R, Ake J, Howard R, Olson S, Paolino K et al. Nephrotoxicity associated with intravenous colistin (colistimethate sodium) treatment at a tertiary care medical center. Clin Infect Dis. 2009; 48:1724-1728.

27. Alothman GA, Ho B, Alsaadi MM, Ho SL, O'Drowsky L, Louca E, et al. Bronchial constriction and inhaled colistin in cystic fibrosis. Chest 2005; 127(2):522-529.

28. https://clinicaltrials.gov/ct2/show/NCT01863719 Downloaded on 8th August 2021

29. Rozenberg-Arska M, Dekker AW, Verhoef J. Colistin and trimethoprim-sulfamethoxazole for the prevention of infection in patients with acute non-lymphocytic leukemia. Decrease in emergence of resistant bacteria. Infection. 1983; 11(3):167-169.

30. Olaitan AO, Morand S, Rolain J-M. Mechanisms of polymyxin resistance: acquired and intrinsic resistance in bacteria. Front. Microbiol. 2014; 5:643. https://doi.org/10.3389/fmicb.2014.00643

31. Bjorn B. Acquired resistance to colistin via chromosomal and plasmid-mediated mechanisms in Klebsiella penumoniae. Infectious Microbes and Diseases 2019; 1(1):10-19.

32. Ghazaeian M, Mokhtari M, Kouchek M, Miri MM, Goharani R, Sistanizad M. A comparative study of high-dose colistin administration for the management of multidrug-resistant gramnegative infections in the ICU. Can J Infect. Dis Medical Microbiology. Volume 2020, article ID 3403520. https:// doi.org/10.1155/2020/3403520

33. Biswas S, Brunel J-M, Dubus J-C, Reynaud-Gaubert M, Rolain JM. Colistin: An update on the antibiotic of the $21^{\text {st }}$ century. Expert Review of Anti-Infective Therapy. 2012; 10(8):917-934.

34. Band VI, Satola SW, Burd EM, Farley MM, Jacob JT, Weiss DS. Carbapenem-resistant klebsiella pneumoniae exhibiting clinically undetected colistin heteroresistance leads to treatment failure in a murine model of infection. mBio. 2018; 9(2):e02448-17. Doi: 10.1128/mBio.02448-17

35. Falagas ME, Sideri G, Vouloumanou EK, Papadatos JH, Kafetzis DA. Intravenous colistimethate (colistin) use in critically ill children without cystic fibrosis. Pediatr Infect Dis J. 2009; 28:123-127.

36. Sahbudak BZ, Kamit CF, Yazici P, Berna AA, Duyu M, Yilmaz CD et al. The evaluation of safety and efficacy of colistin use in pediatric intensive care unit: Results from two reference hospitals and review of literature. J. Infect. Chemother 2018; 24(5):370-375.

37. Tamma PD, Newland JG, Pannaraj PS, Metjian TA, Banerjee R, Gerber JS et al. The Use of Intravenous Colistin Among Children in the United States: Results From a Multicenter, Case Series. Pediatr Infect Dis J. 2013; 32(1):17-22.

38. Karli A, Paksu MS, Karadag A, Belet N, Paksu S, Guney AK et al. Colistin use in pediatric intensive care unit for severe nosocomial infections: experience of a university hospital. Annals of Clinical Microbiology and Antimicrobials. 2013; 12:32. Doi: 10.1186/14760711-12-32.

39. Nation RL, Garonzik SM, Li J, Thamlikitkul V, GiamarellosBourboulis EJ, Paterson DL et al. Updated US and European dose recommendations for intravenous colistin: how do they perform? Clin Infect Dis. 2016; 62:552-558.

40. Nakwan N, Usaha S, Chokephaibulkit K, Villani P, Regazzi M, Imberti R. Pharmacokinetics of colistin following a single dose of intravenous colistimethate sodium in critically ill neonates. Pediatr Infect Dis J. 2016; 35:1211-1214.

41. Nakwan N, Lertpichaluk $P$, Chokephaibulkit K, Villani $P$, Regazzi $\mathrm{M}$, Imberti R. Pulmonary and systemic pharmacokinetics of colistin following a single dose of nebulized colistimethate in mechanically ventilated neonates. Pediatr Infect Dis J. 2015; 34:961-963.

42. Antachopoulos C. Colistin Use in Neonates and Children The Pediatric Infectious Disease Journal 2017; 36(9):905-907.

43. Alan S, Yildiz D, Erdeve O, Cakir U, Kahvecioglu D, Okulu E et al. Efficacy and safety of intravenous colistin in preterm infants with nosocomial sepsis caused by Acinetobacter baumannii. Am J Perinatol. 2014; 31:1079-1086.

44. Serafettin Tekgunduz K, Kara M, Caner I, Demirelli Y. Safety and efficacy of intravenous colistin in neonates with culture proven sepsis. Iran J Pediatr. 2015; 25:e453. DOI: 10.5812/ijp.453. 